\title{
A Child With Acute Liver and Kidney Failure Ongoing Encephalopathy: Effectiveness of Plasmapheresis on Acetaminophen Overdose, A Case Report
}

\author{
Zeliha Haytoglu
}

\begin{abstract}
Acetaminophen (APAP) is a commonly used analgesic and antypiretic medication. APAP-induced liver necrosis has been studied extensively, but extrahepatic manifestations of APAP toxicity are not described well in the literature. Adolescents and young adults may be more prone to renal insufficiency in the setting of APAP overdose; however, the reason for this finding is unclear. Here we report therapeutic misadventure of APAP-associated acute renal and liver failure and effectiveness of plasmapheresis on hepatic encephalopathy.
\end{abstract}

Keywords: Acetaminophen; Nephrotoxicity; Liver failure; Plasmapheresis; Hepatic encephalopathy

\section{Introduction}

Acetaminophen (APAP) is a commonly used over the counter analgesic and major cause of acute liver failure following accidental and intentional overdose [1]. Whereas the occurrence of fulminant hepatic failure is well characterized, there are few reliable data concerning the incidence of APAP-induced nephrotoxicity. Limited data in a retrospective case series of pediatric patients with APAP poisoning suggest that associated nephrotoxicity may be more common in children and adolescents [2]. Acute renal failure occurs in $2-10 \%$ of patients who ingest excessive amounts of paracetamol [3]. Two-thirds of infants and children presenting with acute liver failure develop hepatic encephalopathy (HE) during their first week following initial presentation [4]. In patients who have grade III-IV encephalopathy, $70-80 \%$ patients develop

Manuscript accepted for publication March 31, 2014

Adana Teaching and Medical Research Center, Department of Pediatrics, Baskent University Faculty of Medicine, 01140 Adana, Turkey. Email:zelihahayt@yahoo.com

doi: http://dx.doi.org/10.14740/ijcp143w intracranial hypertension. It is also the most common cause of death in them. Bowel washes, lactulose and anticonvulsants (if seizure present) are the main treatments of HE. Also position of head in neutral position, mechanical ventilation (if Glasgow coma scale $<8$ or respiratory failure presents), hypertonic saline and mannitol are the managements used to treat intracranial hypertension [5]. Recent studies have demonstrated the activation of microglial cells in the brain during liver failure and confirmed a central neuroinflammatory response [6]. In animal models of ischemic or toxic liver injury, microglial activation and concomitantly increased expression of genes coding for proinflammatory cytokines in the brain occur early in the progression of encephalopathy and brain edema [7]. The prevention of proinflammatory process or maybe the cytokines that were activated during acute liver failure can prevent the HE. Here we report a case with acute hepatic, kidney failure and HE-associated paracetamol overdose. Because of ongoing grade III encephalopathy, we performed plasmapheresis. We think plasmapheresis may be effective to reduce cytokines-caused HE, and the improvement seen on HE may be attributable to this effect of plasmapheresis.

\section{Case Report}

A previously healthy, 18-month-old girl with acute liver failure was admitted to our Intensive Care Unit from a state hospital. Seven days before initial admission, fever was observed by her parents. Only runny nose and cough were mainly complaints. On the admission day vomiting and somnolence were observed. Because of ongoing fever, $250 \mathrm{mg}$ APAP, four times 1 day, $110 \mathrm{mg} / \mathrm{kg} /$ day APAP was given to her, for the last 7 days by her parents. On admission she was somnolence. Vital signs were as follows: blood pressure 137/75 mm Hg, pulse 170/min, respiration 45/min. Laboratory findings were as follows: white blood cell count $11,900 / \mathrm{mm}^{3}$, platelet count $287,000 / \mathrm{mm}^{3}$, hemoglobin $9 \mathrm{~g} /$ $\mathrm{dL}, \mathrm{C}$-reactive protein $7 \mathrm{mg} / \mathrm{L}$, ALT 6,851 IU/L, AST 4,202 $\mathrm{IU} / \mathrm{L}$, total bilirubin $0.97 \mathrm{mg} / \mathrm{dL}$, direct bilirubin $0.83 \mathrm{mg} /$ $\mathrm{dL}$, ammonia level $2.93 \mu \mathrm{g} / \mathrm{mL}$, protrombin time $52 / \mathrm{s}$, INR $5 / \mathrm{s}$, glucose $55 \mathrm{mg} / \mathrm{dL}$, BUN $34 \mathrm{mg} / \mathrm{dL}$, creatin $1.4 \mathrm{mg} / \mathrm{dL}$, 
$\mathrm{Na} 137 \mathrm{mEq} / \mathrm{L}$, K $3.5 \mathrm{mEq} / \mathrm{L}$ and paracetamol level $25 \mu \mathrm{g} /$ $\mathrm{mL}$. Viral serology was as follows: $\operatorname{HbsAg}(-)$, antiHBs (-), antiHAVIgM (-), antiHCV (-), CMV IgM (-) EBVIgM and EBVIgG (-). Nasal swab PCR was negative for respiratory viruses. Blood culture was negative. After being transferred to our hospital, she developed seizure. Glasgow coma scale was 7 , and pulse oximetry was $85 \%$. She was intubated and put on mechanical ventilation. On cerebral tomography, brain edema was observed. We diagnosed this clinical picture acute fulminant hepatic failure with grade III encephalopathy associated with APAP overdose. Intravenous N-acetylcysteine (NAC) was started. There was no urine output for $5 \mathrm{~h}$ after admission and hypertension occurred. Creatin level rose from $1.4 \mathrm{mg} / \mathrm{dL}$ to $1.7 \mathrm{mg} / \mathrm{dL}$. We performed peritoneal dialysis on admission day. Protein intake and volume was restricted, and brain edema treatment was started. On second day urinalysis was consistent with acute tubular necrosis (ATN). On fourth admission day, dialysis discontinued.

With medical therapy (reduction protein intake, treated suspected sepsis, removed sedative medication, normal ammonia level, NAC and lactulose), the patient's liver function test subsequently improved but grade III encephalopathy continued. On the fourth admission day and the every other day, plasmapheresis was performed three times. Gradually HE resolved after third plasmapheresis session. She was discharged on day 20 after her admission.

\section{Discussion}

Acute renal failure is a recognized complication of APAP overdose, but has received comparatively little attention. APAP is a known toxin to both the liver and extrahepatic tissues [3]. When significant APAP-induced hepatotoxicity occurs, renal injury is commonly seen. A creatin elevation $>2.0 \mathrm{mg} / \mathrm{dL}$ was noted in $43-57 \%$ of a prospective study of 275 patients with encephalopathy and coagulopathy secondary of APAP-induced hepatic failure [8]. APAP-induced renal insufficiency is consistent with ATN [9]. Urinalysis can be used to differentiate this from other causes of renal insufficiency such as hepatorenal syndrome or prerenal azotemia. In the setting of ATN, urine sediment will have granular casts with variable hematuria or pyuria. In ATN urine sodium tends $>20 \mathrm{mmol} / \mathrm{L}$ with an osmalality similar to that of plasma. In hepatorenal syndrome and prerenal etiologies urine sodium is $<10 \mathrm{mmol} / \mathrm{L}$ and the osmalility is greater than of plasma [10]. In our case there was microscopic hematuria and urine sodium was $>20 \mathrm{mmol} / \mathrm{L}$ consistent with ATN. There were no urine output for $5 \mathrm{~h}$ and hypertension occurred, and we performed periton dialysis. On fourth admission day, dialysis discontinued.

$\mathrm{HE}$ is a neuropsychiatric syndrome associated with hepatic dysfunction. Hyperammonemia is a universally recognized factor in the cascade of events that lead to cerebral edema and intracranial hypertension. Ammonia levels of $>$ $200 \mu \mathrm{M}$ are a strong predictor of development of intracranial hypertension [11]. In our case with medical treatment, ammonia levels were maintained within normal range. In addition markers of inflammation such as necrosis factor alpha (TNF- $\alpha$ ), IL-1 $\beta$ and IL-6 are increased in acute liver failure perhaps reflecting the systemic inflammatory response syndrome (SIRS). Excess in microglia activation seems to be the final pathway for HE-related brain injury. The HE is characterized by cognitive and sleep dysfunction due to overexpression of TNF- $\alpha$ and IL-1 $\beta$ [12-14]. The present understanding is incomplete; the development of intracranial hypertension in patients with acute liver failure seem to be multi-factorial, reflecting brain edema associated with the accumulation of ammonia and cerebral lactic acid and the induction of TNF- $\alpha$ and IL-1 $\beta$. Allowing therapies such as plasmapheresis helps reduction of cytokines because plasmapheresis facilitates the removal of suspected toxins, cytokines, immun-mediated antigens from blood. Larsen et al have previously shown that high volume plasmapheresis can alleviate brain edema in some patients with acute liver failure [15]. Plasmapheresis may also have a positive impact of alleviating the systemic immune dysfunction and endothelial dysfunction that commonly develops. In our case, despite medical treatment for HE continued, after plasmapheresis HE improved gradually. We think plasmapheresis may be effective to reduce cytokines-caused HE, and the improvement seen on HE may be attributable to this effect of plasmapheresis.

\section{Conflict of Interest}

There is no conflict of interest.

\section{References}

1. Bower WA, Johns M, Margolis HS, Williams IT, Bell BP. Population-based surveillance for acute liver failure. Am J Gastroenterol. 2007;102(11):2459-2463.

2. Boutis K, Shannon M. Nephrotoxicity after acute severe acetaminophen poisoning in adolescents. J Toxicol Clin Toxicol. 2001;39(5):441-445.

3. Prescott LF. Paracetamol overdosage. Pharmacological considerations and clinical management. Drugs. 1983;25(3):290-314.

4. Squires RH, Jr., Shneider BL, Bucuvalas J, Alonso E, Sokol RJ, Narkewicz MR, Dhawan A, et al. Acute liver failure in children: the first 348 patients in the pediatric acute liver failure study group. J Pediatr. 2006;148(5):652-658.

5. Bhatia $\mathrm{V}$, Lodha $\mathrm{R}$. Intensive care management of children with acute liver failure. Indian $\mathrm{J}$ Pediatr. 2010;77(11):1288-1295. 
6. Wright G, Shawcross D, Olde Damink SW, Jalan R. Brain cytokine flux in acute liver failure and its relationship with intracranial hypertension. Metab Brain Dis. 2007;22(3-4):375-388.

7. Jiang W, Desjardins P, Butterworth RF. Direct evidence for central proinflammatory mechanisms in rats with experimental acute liver failure: protective effect of hypothermia. J Cereb Blood Flow Metab. 2009;29(5):944952.

8. Larson AM, Polson J, Fontana RJ, Davern TJ, Lalani E, Hynan LS, Reisch JS, et al. Acetaminophen-induced acute liver failure: results of a United States multicenter, prospective study. Hepatology. 2005;42(6):1364-1372.

9. Blantz RC. Acetaminophen: acute and chronic effects on renal function. Am J Kidney Dis. 1996;28(1 Suppl 1):S3-6

10. Mazer M, Perrone J. Acetaminophen-induced nephrotoxicity: pathophysiology, clinical manifestations, and management. J Med Toxicol. 2008;4(1):2-6.
11. Clemmesen JO, Larsen FS, Kondrup J, Hansen BA, Ott P. Cerebral herniation in patients with acute liver failure is correlated with arterial ammonia concentration. Hepatology. 1999;29(3):648-653.

12. Fiore M, Probert L, Kollias G, Akassoglou K, Alleva E, Aloe L. Neurobehavioral alterations in developing transgenic mice expressing TNF-alpha in the brain. Brain Behav Immun. 1996;10(2):126-138.

13. Allan SM, Rothwell NJ. Cytokines and acute neurodegeneration. Nat Rev Neurosci. 2001;2(10):734-744.

14. Petty MA, Lo EH. Junctional complexes of the bloodbrain barrier: permeability changes in neuroinflammation. Prog Neurobiol. 2002;68(5):311-323.

15. Larsen FS, Hansen BA, Ejlersen E, Secher NH, Clemmesen JO, Tygstrup N, Knudsen GM. Cerebral blood flow, oxygen metabolism and transcranial Doppler sonography during high-volume plasmapheresis in fulminant hepatic failure. Eur J Gastroenterol Hepatol. 1996;8(3):261-265. 